\title{
Ueber den Eiweisskörper (fibrinogene Substanz) der vesicula seminalis der Meerschweinchen.
}

\author{
Von
}

\section{H. A. Landwehr,}

Assistent am physiologischen Institut der Universität Kiel.

Bei einigen Thieren folgt dem in die Scheide ergossenen Samen das Secret der glandulae seminales nach, gerinnt hier und bildet so einen das Austliessen des Samens hindernden Versehluss. Herr Prof. Hensen machte mich auf dies Verhalten aufmerksam und forderte mich auf, dasselbe näher zu untersuchen. Wie weit es unter den Thieren verbreitet ist, weiss ich nicht, sehr ausgesprochen ist es bei den Meerschweinchen. Hier scheint der Verschluss für das Zustandekommen der Befruchtung von Bedeutung zu sein. Das Weibchen wird nämlich gleich, nachdem es geworfen hat, belegt. Der Samen gelangt also in die noch weite und blutige Scheide und könnte ohne den Verschluss leicht verloren gehen. Die nachfolgenden Angaben beziehen sich auf das Meerschweinchen.

Bei Eröffnung der Bauchböhle fallen sogleich die ca. $1 \mathrm{dcm}$ langen, an der Wurzel 6-9 $\mathrm{mm}$ dicken, nach der Spitze sich allmählich verjüngenden, drehrunden, mit einigen knorrigen Prominenzen versehenen Samenbläschen in die Augen. Die ca. $1-1^{1 / 2} \mathrm{~mm}$ starke Wand ist aus 3 Schichten zusammengesetzt. Auf die äussere Faserschicht folgt eine $0,3-0,4 \mathrm{~mm}$ dicke Schicht glatter Muskelfasern, welche aus der Länge nach, quer und schief verlaufenden Fasern zusammengesetzt ist. Die innerste $0,7-0,8 \mathrm{~mm}$ dicke Sehicht besteht aus dicht aneinander gereihten, mit Cylinderepithel besetzten Zotten.

Das Secret bildet im normalen Zustande eine wasserklare, schwach opalescirende Gallerte, in der ich mikroskopisch niemals Spermatozoen oder andere morphologische Elemente gefunden habe. An der Luft oder in destillirtem Wasser trübte sich das Secret nach einiger Zeit, blieb aber immer weich, wenn in ersterem Falle 
die Austrocknung durch eine feuchte Kammer vermieden wurde. Mit Blut oder Blutwasser in Bertihrung gebracht, gerann es jedoch sofort und erreichte in kurzer Zeit eine hornartige Härte. Das Secret lässt sich über $80^{\circ}$ erwärmen ohne zu coaguliren. Beim stärkeren :Erhitzen, sowie auch durch Uebergiessen mit Alcohol gerinnt es zu einer harten Masse, die jedoch nicht die zähe, hornartige Consistenz des durch Blut geronnenen Secrets besitzt. Um bei der Entleerung der Driise eine Berührung des Secrets mit Blut zu vermeiden, spritze ich die Gefässe, von der Aorta aus, wiederholt mit Kochsalzlösung aus, wasche die Drüse auch äusserlich ab und schneide sie an, nachdem um die Basis ein Faden geschnürt war.

Das Secret zeigt eine verschiedene Concentration. In einem ziemlich concentrirten fand ich $71 \%$ Wasser und $29 \%$ eines Eiweisskörpers, der, wie sein Verhalten zu Salzlösungen and verdünnten Säuren zeigt, den Globulinen zuzurechnen ist. $1,262 \mathrm{gr}$ Secret mit $29 \%$ fester Substanz liessen nach dem Verbrennen keine wägbare Aschenmenge zurïck.

Der Eiweisskörper giebt mit Kupfersulfat und Natronlauge eine violette Färbung; zeigt die Xanthoproteinreaction und färbt sich durch Millonsches Reagens roth. Durch verdünnte Chlorwasserstoffsäure $(1 \%)$ wird er leicht gelöst und in Syntonin iibergeführt. Die Lösung zeigt folgende Eigenschaften:

Sie wird durch Kochen nicht getrübt. Genaues Neutralisiren fällt das Syntonin, welches, wie alle Syntonine, in sehr verdünnten kohlensauren Alkalien leicht löslich ist. Die Lösung wird nicht getribt auf Zusatz von cone. Mineralsäuren, auch nicht auf Hinzufügung von cone. Kochsalzlösung.

Eintragen von Kochsalzkrystallen fällt das Eiweiss aus seiner Lösung. Auf Alcoholzusatz bleibt die Lösung klar, Aether fällt aber die alcoholisehe Lösung.

Das Secret wird gelöst durch Kochsalzlösungen. Bittersalzund Clorealciumlösungen nehmen es auch auf. Die günstigste Concentration derselben ist für verschiedene Temperaturen verschieden. Erwärmen trübt eine gesättigte $1 / 2 \%$ ige NaCl-lösung, Kälte eine solche, die $15-20 \% \mathrm{Na} \mathrm{Cl}$ enthält. Für mittlere Temperaturen zeigt sich eine $8 \%$ ige Lösung am günstigsten. Diese zeigt das folgende Verhalten: Sie wird gefällt durch $\mathrm{NH}_{3},\left(\mathrm{NH}_{4}\right)_{2} \mathrm{CO}_{3}, \mathrm{NaOH}$, oder $\mathrm{Na}_{2} \mathrm{CO}_{3}$, wenig Eiweiss enthaltende Lösungen werden nicht getrïbt. Essigsäure trübt die gesättigte Lösung, aber nicht wenig 
Eiweiss enthaltende Lösungen; erstere wird durch Erhitzen oder Verdünnen wieder klar.

Kochsalzkrystalle fällen den Globulinkörper vollständig aus. Alcohol fällt neben $\mathrm{NaCl}$ auch Albumin; beim Verdïnnen löst sich der Niederschlag und fällt auf erneuten Alcoholzusatz wieder aus. Verdünnung der Globulinlösung mit Wasser trübt sie nicht. $\mathrm{CO}_{2}$ durch die verdünnte Lösung geleitet, triibt sie kaum merklich.

Kalkwasser löst geringe Mengen der Globulinsubstanz. Die Lösung trïbt sich beim Erwärmen auf $55^{\circ}$, und der Niederschlag bleibt auch kalt ungelöst. Die Lösung in $\mathrm{NaCl}$ gerinnt nicht beim Erwärmen. Das Globulin verträgt in der Hitze sogar mehr CINa als in der Kälte. Durch ClNa eben getrübte Lösungen wurden beim Erwärmen wieder klar und trübten sich wieder beim Erkalten. Setzte ich der CINa-lösung jedoch etwas Kalkwasser zu, so gerann sie um $56^{\circ} \mathrm{C}$. herum. Auch eine Lösung des Secrets in $5 \%$ $\mathrm{Cl}_{2} \mathrm{Ca}$ gerann erst auf Zusatz von Kalkwasser.

Nach Hammarsten wird eine Paraglobulinlösung durch Kochsalz nie vollständig gefällt, wohl aber eine Fibrinogenlösung, während letztere, sowohl durch Verdïnnen als auch durch verdünnte Säuren schwieriger gefällt wird als das Paraglobulin. Während eine Paraglobulinlösung, welche weniger als $5 \% \mathrm{NaCl}$ enthält, je nach der Geschwindigkeit des Erwärmens erst bei $69-$ $76^{\circ} \mathrm{C}$. gerinnt, tritt in einer Fibrinogenlösung von demselben $\mathrm{NaCl}$ Gehalt die Gerinnung schon bei $52^{\circ}-55^{\circ} \mathrm{C}$. ein. Sollte die fibrinogene Substanz des Blutes ihre Gerinnbarkeit Spuren von Kalk verdanken?

Das Secret löst sich leicht in verdïnnter Natronlauge $(0,5 \%)$. Auch diese Lösung bleibt beim Kochen klar. Durch Alcohol wird sie nicht getrübt, wohl aber durch Aether die alcoholische Lösung. Kochsalz in Substanz fällt die Lösung.

Eine Lösung in verdünnter Sodalösung verhält sich ebenso.

Im reinen Zustande sind Globulinkörper bisher nicht bekannt gewesen, alle sind - die Fibrin bildenden nicht ausgenommen mindestens lecithinhaltig. Aus dem Samenblasensecret konnte weder durch Alcohol noch durch Aether Lecithin oder überhaupt etwas extrahirt werden.

Bringt man einen längeren Streifen des Drïsensecretes in eine Schale und berührt das eine Ende desselben mit etwas Blutwasser, so trübt sich der 'ganze Streifen sogleich und wird nach 
Ueber d. Eiweisskörper (fibrinogene Substanz) der vesicula seminalis etc. 541

einigen Secunden fest. Das Blutwasser scheint dabei nur eine katalytische Wirkung zu haben; denn es dringt nichts von demselben in das Secret ein, und das rein weisse Fibrin ist scharf gegen den rothen Punkt abgegrenzt. Das so erhaltene Fibrin verhält sich ganz wie Blutfibrin. In verdünnten Säuren quillt es auf und geht theilweise in Lösung über, je frischer es ist, desto mehr. Um es in Syntonin iiberzufïhren, verfährt man ganz wie beim Blutfibrin. Es wird mit rauchender Salzsäure behandelt bis zum Rothwerden der letzteren. Verdünnt man die abgegossene Salzsäure mit dem Vierfachen an Wasser, so fällt das Syntonin in Flocken aus. Die Wirkung des Blutwassers ist wahrscheinlich dem Fibrinferment zuzuschreiben. Leider habe ich keine Fermentlösung zur Hand, um dies za entscheiden. Aeussere Umstände, sowie die für solche Arbeiten höchst ungïnstige warme Witterung zwingen mich, die weitere Untersuchung vorläufig ruhen zu lassen.

Bischoff und Andere haben des Scheidenverschlusses durch ein Secret der vesicula seminalis schon Erwähnung gethan. Hensen $\left.{ }^{1}\right)$ giebt zuerst Reactionen der Masse des Scheidenpfropfes an. $\mathrm{Er}$ findet dieselbe in conc. $\mathrm{H}_{2} \mathrm{SO}_{4}$ und in $\mathrm{NaOH}$ schwer löslich, in Kochsalzlösungen quellend - Eigenschaften; wie sie auch das Blatfibrin einige Zeit nach der Gerinnung annimmt.

Das Verhalten dieses dem Fibrinogen so sehr ähnlichen Körpers spricht sehr dafür, dass das Paraglobulin keine wesentliche Rolle bei der Blutgerinnung spielt.

Zum Schluss sage ich Herrn Prof. Hensen für die der Arbeit gewährte Unterstützung meinen besten Dank.

1) Zeitschrift für Anat. und Entwicklungsgesch. 1876. 